\title{
Differentiation between Tuberculous Spondylitis and Pyogenic Spondylitis on MR Imaging
}

\author{
Jong-Han Park', Hye-Seon Shin ${ }^{2}$, Jong Tae Park', Tae Young Kim', Ki Seong Eom ${ }^{1}$ \\ ${ }^{1}$ Departments of Neurosurgery, ${ }^{2}$ Radilogy, Wonkwang University School of Medicine, Iksan, Korea
}

Objective: The objective of this study was to compare the magnetic resonance (MR) imaging of tuberculous spondylitis with pyogenic spondylitis.

Methods: MR images of the spines of 41 patients with infectious spondylitis at our institution over 8-years of period were retrospectively reviewed. Eighteen patients with infective spondylitis were excluded because their results on the marrow biopsy and culture were negative. MR imaging findings in 6 patients with tuberculous spondylitis ( 3 male, 3 female) were compared with those of 17 patients (10 male, 7 female) with pyogenic spondylitis.

Results: Two MR imaging findings were statiscally significant in differentiating the tuberculous spondylitis from pyogenic spondylitis: a well defined paraspinal abnormal signal and a thin and smooth abscess wall. There were no significant differences in the following MR imaging findings: paraspinal abscess or intraosseous abscess, subligamentous spread to three or more vertebra, involvement of multiple vertebra, hyperintense signal on T2-weighted images, heterogenous low signal on T1-weighted images, involvement of posterior element, epidural extension, involvement of intervertebral disk, disk space narrowing, rim enhancement of the abscess, skip lesion, and endplate destruction.

Conclusion: MR imaging is an appropriate modality for differentiation of tuberculous spondylitis from pyogenic spondylitis.

Key Words: Spondylitis · Tuberculous $•$ Pyogenic $\cdot$ MR Imaging

\section{INTRODUCTION}

Infectious spondylitis is defined as an infection by a specific organism of one or more components of the spine, namely the vertebra, intervertebral discs, paraspinal soft tissues, and epidural space ${ }^{16}$. Infectious spondylitis represents $2-4 \%$ of all cases of osteomyelitis. Males are affected more frequently than females (ratio of 2 to 1), usually in the fifth to sixth decades, but it may appear at any age ${ }^{17}$. Although infection can be caused by pyogenic, granulomatous, autoimmune, idiopathic, and iatrogenic conditions, pyogenic spondylitis is the most common spinal infection, and tuberculosis continues to be a major problem in developing countries and is resurgent in Western world with the onset of human immunodeficiency virus $(\mathrm{HIV})^{13)}$.

The clinical differentiation of pyogenic and tuberculous spondylitis is important because the medications used to man-

- Received: November 18, 2011 • Revised: December 29, 2011

- Accepted: January 9, 2012

Corresponding Author: Ki Seong Eom, MD, PhD

Department of Neurosurgery, Wonkwang University School of Medicine,

344-2 Shinyong-dong, Iksan 570-749, Korea

Tel: +82-63-859-1467; Fax: +82-63-852-2606.

E-mai: kseom@wonkwang.ac.kr age these infections are notably different. Early diagnosis and prompt treatment are essential to prevent permanent neurologic deficit and spinal deformity ${ }^{5,11}$. However, it is difficult to differentiate tuberculous spondylitis from pyogenic spondylitis clinically and radiolgically ${ }^{3,16}$. Magnetic resonance (MR) imaging has been useful in the detection of spinal disorders, and its high sensitivity in detecting early changes has been valuable $^{12)}$. The purpose of our study was to evaluate the MR imaging feature differentiating tuberculous spondylitis from pyogenic spondylitis.

\section{MATERIAL AND METHODS}

\section{Patient population}

MR images of the spines of 41 patients with infectious spondylitis at our institution over 8-years of period were retrospectively reviewed. After the medical record review, $18 \mathrm{pa}-$ tients with infective spondylitis were excluded because their results on the marrow biopsy and culture were negative. All 17 cases of pyogenic spondylitis and 6 cases of tuberculous spondylitis were confirmed by biopsy and culture. The patient comprised 17 with pyogenic spondylitis (10 male, 7 female) 
and 6 with tuberculous spondylitis ( 3 male, 3 female). The mean age of the patient with pyogenic spondylitis and tuberculous spondylitis was 63.6 years (range 51-78 years) and 49.2 years (range 29-74 years), respectively. The mean interval from presentation to MR imaging was 7 weeks (range 1-14 weeks) in patients with pyogenic spondylitis and 26 weeks (range 1 week - 24 months) in patients with tuberculous spondylitis.

\section{MR imaging}

MR imaging was performed on 1.5-T imager (Intera, Philips Medical Systems), using a surface coil or spine coil. Axial and sagittal T1-weighted MR images and fast spin-echo or turbo spin-echo T2-weighted images were obtained. In addition, axial, sagittal and coronal fat-suppressed T1-weighted images were obtained after IV infusion of $0.1 \mathrm{mmol} / \mathrm{kg}$ of dimeglumine gadopentetate. Typical MR parameters were as follows: field of view, $140-280 \mathrm{~mm}$ for axial plane, $300 \mathrm{~mm}$ for sagittal plane and $344 \mathrm{~mm}$ for sagittal plane; number of excitations, 2; slice thickness, $4 \mathrm{~mm}$ and intersection gap, $4 \mathrm{~mm}$.

\section{Statistical analysis}

Statistical analysis was performed with independent twosample T-test and ANOVA (Analysis Of Variance).

\section{RESULTS}

The tuberculous spondylitis (Fig. 1) group consisted of 3 males (50\%) and 3 females (50\%). Whereas, the pyogenic spondylitis (Fig. 2) group consisted of 10 males (58.8\%) and 7 females $(41.2 \%)$. The location that each spondylitis is involved is summarized in Table 1 . Thoracic spine was the most common

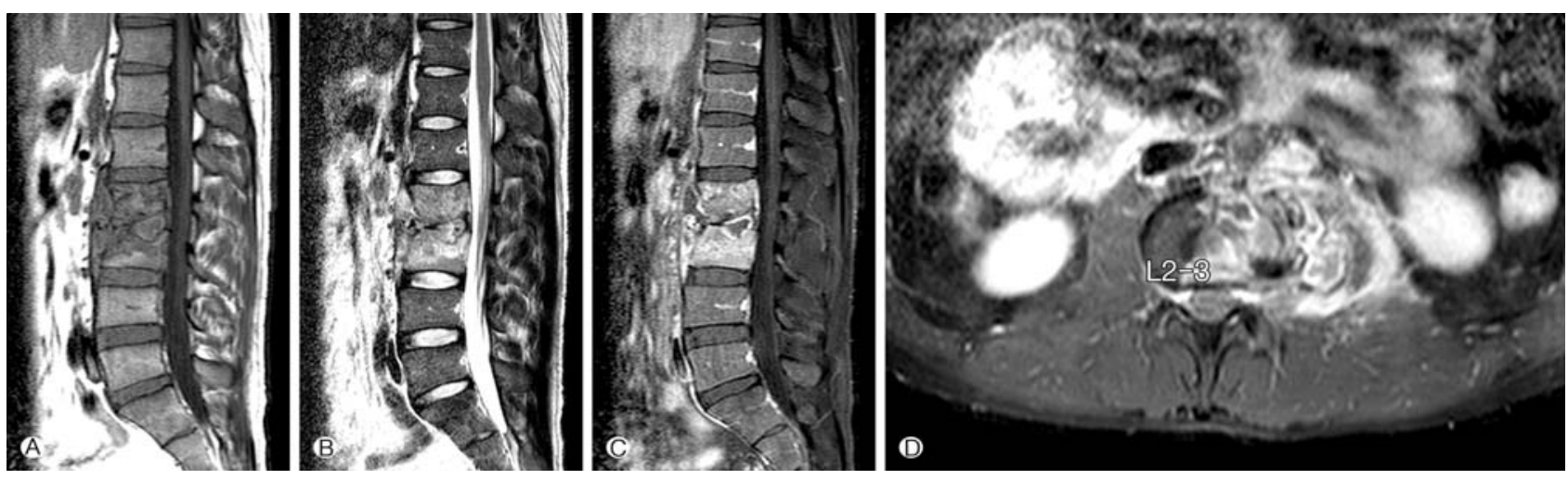

Fig. 1. A 27-year old male with tuberculous spondylitis. A: T1-weighted image (T1WI) shows heterogeneously hypointense signal in L2-3 vertebral bodies and subligamentous spread. B: T2WI shows L2-3 inhomogeneous hyperintense signal. C: Fat-suppressed contrast-enhanced TIWI shows rim enhancing intervertebral disk of L2-3. D: Axial fat-suppressed contrast-enhanced T1WI shows thin and smooth rim enhancement of paraspinal abscess.
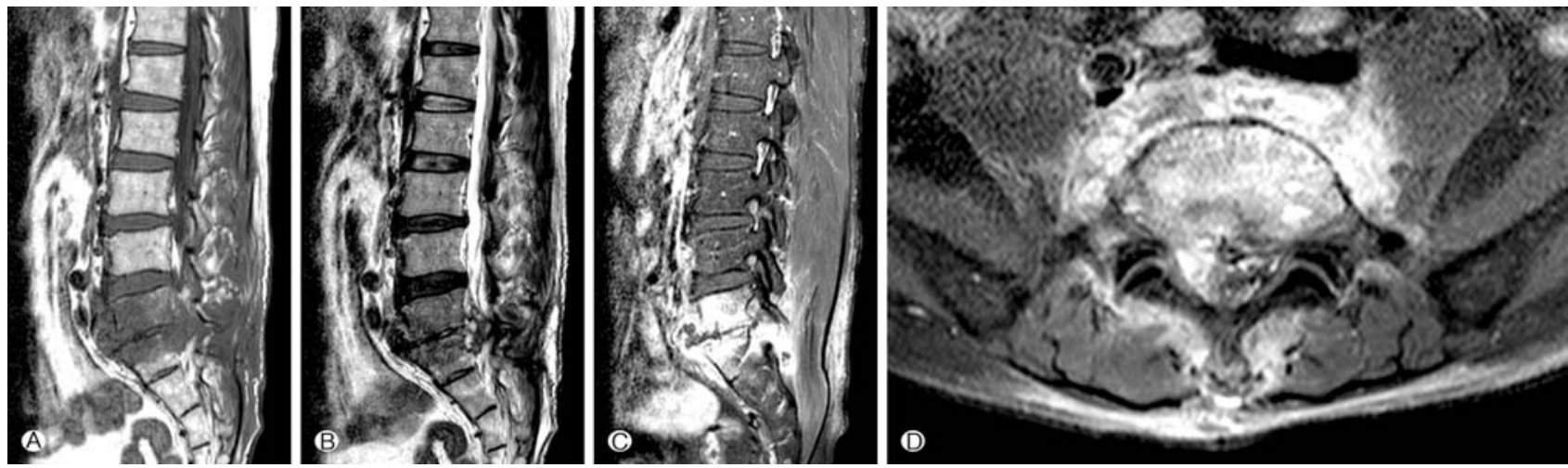

Fig. 2. A 55 year-old male with pyogenic spondylitis. A: T1-weighted image (T1WI) shows hypointensity of L5-S1 vertebral bodies. B: T2WI shows isointese to hypointense singal of L5-S1 vertebral bodies. C: Fat-suppressed contrast-enhanced T1WI shows diffuse heterogenous enhancement in the L5-S1 vertebral bodies. D: Axial fat-suppressed contrast-enhanced T-weighted image shows illdefined paraspinal enhancement. Thick and irregular rim enhancement of paraspinal abscess is present. 
site of tuberculous spondylitis involvement.

The mean affected verterbra number was 2.67 (range 2-4) in tuberculous spondylitis and 2.88 (range 2-4) in pyogenic spondylitis. The results of the imaging findings for tuberculous spondylitis and pyogenic spondylits are summarized in Table 2.

The incidence of the following MR imaging findings was singnificantly higher in patients with tuberculous spondylitis than in those with pyogenic spondylitis $(\mathrm{p}<0.05)$ : a well defined paraspinal abnormal signal ( $83 \%$ in tuberculous vs $41 \%$ in pyogenic) and a thin and smooth abscess wall (83\% vs $24 \%$ ). There were no significant differences in the following MR imaging findings : paraspinal abscess or intraosseous abscess ( $100 \%$ in tuberculous vs $71 \%$ in pyogenic, $\mathrm{p}=0.146)$, subligamentous spread to three or more vertebra (50\% vs $12 \%$, $\mathrm{p}=0.054)$, involvement of multiple vertebra ( $33 \%$ vs $53 \%$, $\mathrm{p}=0.432)$, hyperintense signal on T2-weighted images $(67 \%$ vs $65 \%, \mathrm{p}=0.935)$, heterogenous low signal on T1-weighted images ( $83 \%$ vs $88 \%, \mathrm{p}=0.772$ ), involvement of posterior element ( $17 \%$ vs $29 \%, \mathrm{p}=0.589)$, epidural extension $(100 \%$ vs $76 \%, \mathrm{p}=0.208)$, involvement of intervertebral disk ( $83 \%$ vs $82 \%, \mathrm{p}=0.959)$, disk space narrowing ( $50 \%$ vs $35 \%, \mathrm{p}=0.547$ ), rim enhancement of the abscess ( $67 \%$ vs $35 \%, p=0.199)$, skip lesion ( $33 \%$ vs $12 \%, \mathrm{p}=0.250)$, and endplate destruction $(100$

Table 1. Site of spinal involvement

\begin{tabular}{lcc}
\hline \hline Location & Tuberculous $(\mathrm{n}=6)$ & Pyogenic $(\mathrm{n}=17)$ \\
\hline Cervical spine & $0(0 \%)$ & $1(5.9 \%)$ \\
Thoracic spine & $3(50 \%)$ & $5(29.4 \%)$ \\
Lumbar spine & $1(16.7 \%)$ & $8(47.1 \%)$ \\
Sacral spine & $2(33.3 \%)$ & $3(17.6 \%)$ \\
\hline
\end{tabular}

$\%$ vs $71 \%, \mathrm{p}=0.146)$.

\section{DISCUSSION}

The incidence of typical acute vertebral osteomyelitis has decreased because of the more wide spread use of antibiotics. However, tuberculous spondylitis is still a frequent cause of infectious spondylitis in endemic regions and is increasing in prevalence because of the resurgence of tuberculosis during the past decades, especially in patients who are immunocompromised $^{3,13,15,16}$.

The clinical differentiation of pyogenic and tuberculous spondylitis is important because the medications are definitely different $^{6}$. However, differentiation between tuberculous and pyogenic spondylitis is difficult clinically and radiographically ${ }^{12)}$. MR imaging has been reported to be useful in the early detection of spondylitis ${ }^{1,3,9,20)}$. There have been several reports about the differences on MR imaging findings between tuberculous spondylitis and pyogenic spondylitis. We also studied on the differences on MR imaging findings. We demonstrated that the two MR imaging findings, a well defined paraspinal abnormal signal $(83 \%$ in tuberculous vs $41 \%$ in pyogenic) and a thin and smooth abscess wall (83\% vs $24 \%$ ) are reliable suggesting tuberculous spondylitis in our study. Our study is corresponding to Jung et al. ${ }^{12)}$ The smoother margin of the paraspinal abnormal signal and a thinner and smoother abscess wall are due to the relative late phase and chronic course of tuberculous spondylitis ${ }^{12}$. The minimal inflammation of tuberculous abscess also might be the reason to the thin and smooth appearance of abscess wall ${ }^{12)}$. Rim enhancement of abscess on MR imaging suggested the tuberculous

Table 2. MR imaging findings of tuberculous spondylitis and pyogenic spondylitis

\begin{tabular}{lccc}
\hline \hline MR imaging findings & Tuberculous spondylitis $(\mathrm{n}=6)$ & Pyogenic spondylitis $(\mathrm{n}=17)$ & $\mathrm{p}$-value \\
\hline Well-defined paraspinal abnormal signal & $83(5)$ & $41(7)$ & 0.017 \\
Thin \& smooth abscess wall & $83(5)$ & $24(4)$ & 0.008 \\
Paraspinal abscess or intraosseous abscess & $100(6)$ & $71(12)$ & 0.146 \\
Subligamentous spread to three or more vertebra & $50(3)$ & $12(2)$ & 0.054 \\
Involvement of multiple vertebra & $33(2)$ & $53(9)$ & 0.432 \\
Hyperintense signal on T2 & $67(4)$ & $65(11)$ & 0.935 \\
Heterogenous low signal on T1 & $83(5)$ & $88(15)$ & 0.772 \\
Involvement of post. element & $17(1)$ & $29(5)$ & 0.589 \\
Epidural extension & $100(6)$ & $76(13)$ & 0.208 \\
Involvement of intervertebral disk & $83(5)$ & $82(14)$ & 0.959 \\
Disk space narrowing & $50(3)$ & $35(6)$ & 0.547 \\
Rim enhancement of the abscess & $67(4)$ & $35(6)$ & 0.199 \\
Skip lesion & $33(2)$ & $12(2)$ & 0.250 \\
Endplate destruction & $100(6)$ & $71(12)$ & 0.146 \\
\hline
\end{tabular}

Values are shown as \% (no. of cases). 
spondylitis by other reports ${ }^{1,3,15,16,21)}$. However, rim enhancement (67\% in tuberculous vs $35 \%$ in pyogenic) in our study had no stastically singnificant difference $(p=0.199)$. This finding is supported by Jung et al. 12).

Lack of proteolytic enzymes in Mycobacterium as compared with pyogenic agents of infection has been proposed as the cause of the relative preservation of the intervertebral disks $^{3,8,18)}$. However, the results of our study showed disk space narrowing in 50\% of patients with tuberculous spondylitis and $35 \%$ of those with pyogenic spondylitis. This difference is not statistically significant $(\mathrm{p}=0.547)$. We assume that the disk space narrowing might be attributable to other factors such as a lumbar disk degeneration ${ }^{10)}$.

Subligamentous spread to three or more vertebral levels in tuberculous spondylitis is frequent ${ }^{9,21)}$. Infection may extend from an anterior lesion of the vertebral body beneath the anterior longitudinal ligament to the anterior parts of neighboring intervertebral disk and vertebral bodies ${ }^{10)}$. However, in our study, subligamentous spread was observed in $50 \%$ of patients with tuberculous spondylitis as compared with $12 \%$ of those with pyogenic spondylitis. There was no statistically difference $(p=0.054)$. These discrepancies might be due to the small number of patients in our study. Involvement of the posterior element has been reported in tuberculous spondylitis and very uncommonly in pyogenic spondylitis ${ }^{3,15,18)}$. Our study findings were not corresponding to these previous report, $17 \%$ in tuberculous vs $29 \%$ in pyogenic spondylitis, especially statistically no difference $(\mathrm{p}=0.589)$.

Chang et al. ${ }^{6}$ reported distinguishing the tuberculous spondylitis and pyogenic spondylitis was difficult by using only T2weighted images. Like Chang et al. ${ }^{6}$, Our study findings are no differences between the two groups in T2 signal intensity. There are several limitations associated with this study reported here. The sample size was small. Especially, the number of the patients with tuberculous spondylitis was six, because tuberculosis has been reduced in developed countries in the past 30 years, as a consequence of a precocious diagnosis and mostly with anti-bacillary therapeutics ${ }^{2}$. Tuberculosis of spine also accounts for $1 \%$ of all tuberculosis infection ${ }^{21)}$. Also rates for isolating the causative organism are less than $50 \%$ in tuberculous spondylitis and $60-80 \%$ for pyogenic spondylitis ${ }^{4,7,14}$. Morever, histopathologic evaluation of biopsy material does not always provide a specific diagnosis ${ }^{19,21)}$.

\section{CONCLUSION}

In conclusion, MR imaging is very helpful method for differentiation of tuberculous spondylitis from pyogenic spondylitis. Well defined paraspinal abnormal signal and thin and smooth abscess wall were more suggestive of tuberculous spondylitis than of pyogenic spondylitis.

\section{REFERENCES}

1. Akman S, Sirvanci M, Talu U, Gogus A, Hamzaoglu A: Magnetic resonance imaging of tuberculous spondylitis. Orthopedics 26:69-73, 2003

2. Almeida A: Tuberculosis of the spine and spinal cord. Eur J Radiol 55:193-201, 2005

3. Arizono T, Oga M, Shiota E, Honda K, Sugioka Y: Differentiation of vertebral osteomyelitis and tuberculous spondylitis by magnetic resonance imaging. Int Orthop 19:319-322, 1995

4. Armstrong P, Chalmers A, Green G, Irving J: Needle aspiration/biopsy of the spine in suspected disc space infection. $\mathrm{Br}$ J Radiol 51:333-337, 1978

5. Chan WH, Liu JS, Howng SL: Tuberculous spondylitis: a clinical analysis. Gaoxiong Yi Xue Ke Xue Za Zhi 6:428-434, 1990

6. Chang MC, Wu HTH, Lee CH, Liu CL, Chen TH: Tuberculous spondylitis and pyogenic spondylitis: comparative magnetic resonance imaging features. Spine 31:782-788, 2006.

7. Garcia A Jr, Grantham SA: Hematogenous pyogenic vertebral osteomyelitis. J Bone Joint Surg Am 42-A:429-436, 1960

8. Gouliamos A, Kehagias DT, Lahanis S, Athanassopoulou AA, Moulopoulou ES, Kalovidouris AA, et al: MR imaging of tuberculous vertebral osteomyelitis: pictorial review. Eur Radiol 11:575-579, 2001

9. Griffith JF, Kumta SM, Leung PC, Cheng JCY, Chow LTC, Metreweli C: Imaging of musculoskeletal tuberculosis: a new look at an old disease. Clin Orthop Relat Res 398:32-9, 2002

10. Harada Y, Tokuda O, Matsunaga N: Magnetic resonance imaging characteristics of tuberculous spondylitis vs. pyogenic spondylitis. Clin Imaging 32:303-309, 2008

11. Jaffe HL: Metabolic, degenerative, and inflammatory diseases of bones and joints, vol. 1: Lea \& Febiger Philadelphia; 1972.

12. Jung NY, Jee WH, Ha KY, Park CK, Byun JY: Discrimination of tuberculous spondylitis from pyogenic spondylitis on MRI. Am J Roentgenol 182:1405-1410, 2004

13. Moore SL, Rafii M: Imaging of musculoskeletal and spinal tuberculosis. Radiol Clin North Am 39:329-342, 2001

14. Sapico FL, Montgomerie JZ: Pyogenic vertebral osteomyelitis: report of nine cases and review of the literature. Rev Infect Dis 1:754-776, 1979

15. Shanley DJ: Tuberculosis of the spine: imaging features. Am J Roentgenol 164:659-664, 1995

16. Sharif HS, Morgan JL, Al Shahed MS, Al Thagafi M: Role of CT and MR imaging in the management of tuberculous spondylitis. Radiol Clin North Am 33:787-804, 1995

17. Smith A, Blaser S: Infectious and inflammatory processes of the spine. Radiol Clin North Am 29:809-827, 1991

18. Smith AS, Weinstein MA, Mizushima A, Coughlin B, Hayden 
S, Lakin M, et al: MR imaging characteristics of tuberculous spondylitis vs vertebral osteomyelitis. Am J Roentgenol 153: 399-405, 1989

19. Smith F, Runge V, Permezel M, Smith C: Nuclear magnetic resonance (NMR) imaging in the diagnosis of spinal osteomye- litis. Magn Reson Imaging 2:53-56, 1984

20. Stabler A, Reiser MF: Imaging of spinal infection. Radiol Clin North Am 39:115-135, 2001

21. Thrush A, Enzmann D: MR imaging of infectious spondylitis. Am J Neuroradiol 11:1171-1180, 1990 